\title{
INVOLUTIONS WITH NONZERO ARF INVARIANT
}

\author{
BY ISRAEL, BERSTEIN ${ }^{1}$
}

Communicated by F. P. Peterson, February 26, 1968

Browder and Livesay [1] have associated with each differentiable fixed point free involution $T: \Sigma^{2 q+1} \rightarrow \Sigma^{2 q+1}$, where $\Sigma=\Sigma^{2 q+1}$ is a homotopy sphere, a "signature" $\sigma(\Sigma, T) \in Z(\equiv 0 \bmod 8)$ if $q$ is odd, or an "Arf invariant" $c(\Sigma, T) \in Z_{2}$, if $q$ is even. If $q \geqq 3$, then $\Sigma$ contains a differentiably imbedded $2 q$-sphere invariant with respect to $T$ if and only if $\sigma(\Sigma, T)=0$ or $c(\Sigma, T)=0[1]$.

S. López de Medrano has constructed for every odd $q$ examples of involutions $T$ with nonzero signature. We prove the following

ThEOREM. For every $k \geqq 1$ there exists a fixed point free differentiable involution $T: \Sigma^{4 k+1} \rightarrow \Sigma^{4 k+1}$ with $c(\Sigma, T) \neq 0$. Here $\Sigma$ is the "Kervaire homotopy sphere," i.e., the generator of $b P_{4 k+2}$ [3], [4] if the latter group is $\neq 0$; otherwise it is the standard sphere.

The author understands that D. Montgomery and C. T. Yang have an example of a differentiable involution on $\Sigma^{9}$ with $c(\Sigma, T) \neq 0$. The fact that there are PL-involutions with $c(\Sigma, T) \neq 0$ on any $(4 k+1)$-dimensional sphere follows from the classification of C. T. C. Wall [8].

I would like to thank G. R. Livesay for valuable suggestions and for many discussions which have helped me understand the problem.

1. Recall of definitions. Let $T$ be a differentiable (or PL) fixed point free involution on $\Sigma=\Sigma^{4 k+1}$. A characteristic manifold $N^{4 k}$ is an invariant submanifold such that $\Sigma=A \cup B, N=A \cap B, B=T A$. There always exists such an $N$ which is $(2 k-1)$-connected [1]. Let $G=H_{2 k}\left(N, Z_{2}\right)=H_{2 k}(N) \otimes Z_{2}$. For $x, y \in G$, the intersection coefficients $A(x, y)=x \cdot y \in Z_{2}$, and $B(x, y)=x \cdot T y$ define nonsingular symmetric bilinear forms on $G$ and

$$
A(x, T y)=B(x, y) .
$$

Browder and Livesay [1] define a quadratic form $\psi_{0}: G \rightarrow Z_{2}$ (if $x \in G$ is represented by an immersed sphere $\sigma$ in general position with respect to $T \sigma$, then $\psi_{0}(x)$ is 1 if and only if $\sigma \cap T \sigma$ consists of an odd number of pairs of points). One can also define [7] another qua-

\footnotetext{
${ }^{1}$ Partially supported by NSF Grant GP 3685.
} 
dratic form $\mu_{0}: G \rightarrow Z_{2}$ by $\mu_{0}(x \otimes 1)=\frac{1}{2} x \cdot x \bmod 2$, where $x \in H_{2 k}(N, Z)$. $(x \cdot x$ is always even). We have [1], [7]

$$
\begin{gathered}
\psi_{0}(x)=\psi_{0}(T x), \quad \mu_{0}(x)=\mu_{0}(T x) ; \\
\psi_{0}(x)=0
\end{gathered}
$$

if $x$ can be represented by an immersed sphere $\sigma$ disjoint from $T \sigma$;

$$
\begin{aligned}
& \psi_{0}(x+y)=\psi_{0}(x)+\psi_{0}(y)+B(x, y), \\
& \mu_{0}(x+y)=\mu_{0}(x)+\mu_{0}(y)+A(x, y) .
\end{aligned}
$$

Let $G_{T}=G /(1+T) G$. By (1) and (2), we can define following [7], a pairing $C: G_{T} \otimes G_{T} \rightarrow Z_{2}$ and a quadratic form $\mu_{T}: G_{T} \rightarrow Z_{2}$ by setting

$$
C(\bar{x}, \bar{y})=A(x, y)+B(x, y), \quad \mu_{T}(\bar{x})=\mu_{0}(x)+\psi_{0}(x),
$$

where $x, y \in G$ represent $\bar{x}, \bar{y} \in G_{T}$. Clearly

$$
\mu_{T}(\bar{x}+\bar{y})=\mu_{T}(\bar{x})+\mu_{T}(\bar{y})+C(\bar{x}, \bar{y}) .
$$

Let $i_{A}: G=H_{2 k}\left(N, Z_{2}\right) \rightarrow H_{2 k}\left(A, Z_{2}\right), i_{B}: G \rightarrow H_{2 k}\left(B, Z_{2}\right)$. Then $G=\operatorname{Ker} i_{A} \oplus \operatorname{Ker} i_{B}$.

$$
T \operatorname{Ker} i_{A}=\operatorname{Ker} i_{B}
$$

and (see [1])

$$
\begin{gathered}
B \text { is nonsingular on } \operatorname{Ker} i_{A}, \\
A(x, y)=0 \quad \text { for } x, y \in \operatorname{Ker} i_{A} .
\end{gathered}
$$

Moreover,

$$
\mu_{0}(x)=0 \text { if } x \in \operatorname{Ker} i_{A},
$$

since $x=y \otimes 1, y \in \operatorname{Ker}\left(H_{2 k}(N, Z) \rightarrow H_{2 k}(A, Z)\right)$ and $y \cdot y=0$. By $(7)$ Ker $i_{A}$ maps isomorphically onto $G_{T}$ and by (8) and ( $\left.8^{\prime}\right) C$ is nonsingular. Let $e_{1}, \cdots, e_{n}, f_{1}, \cdots, f_{n} \in \operatorname{Ker} i_{A}$ be a symplectic basis for $B \mid \operatorname{Ker} i_{A}$. Then $\bar{e}_{1}, \cdots, \bar{e}_{n}, \bar{f}_{1}, \cdots, \bar{f}_{n} \in G_{T}$ form a symplectic basis for $C$ and (5) and (9) imply

$$
c(\Sigma, T)=\Sigma_{i} \psi_{0}\left(e_{i}\right) \psi_{0}\left(f_{i}\right)=c_{T}=\Sigma_{i} \mu_{T}\left(\bar{e}_{i}\right) \mu_{T}\left(\bar{f}_{i}\right) .
$$

The advantage of the identity $c(\Sigma, T)=c_{T}$ is that $c_{T}$ is independent of the choice of the symplectic basis $\tilde{e}_{i}, \bar{f}_{i} \in G_{T}$ [7], whereas $c(\Sigma, T)$ is independent of the choice of the characteristic submanifold $N$ [1].

2. The involution $T$. We shall follow here a construction described in [6]. Let $S^{2 k+1} \subset R^{2 k+1}$ be the unit sphere $\|x\|=1$, where $x$ $=\left(x_{0}, x, \cdots, x_{2 k+1}\right)$ and let $S^{2 k} \subset S^{2 k+1}$ be the "meridian" $x_{2 k+1}=0$. Define the rotation $\rho: S^{2 k+1} \rightarrow S^{9 k+1}$ 


$$
\rho\left(x_{0}, x_{1}, \cdots, x_{2 k+1}\right)=\left(x_{0},-x_{1}, \cdots,-x_{2 k+1}\right)
$$

with fixed points $P=(1,0, \cdots, 0)$ and $Q=(-1,0, \cdots, 0) ; \rho\left(S^{2 k}\right)$ $=S^{2 k}$. Let $\lambda:[-1,1] \rightarrow[0,1]=I$ be a $C^{\infty}$ function such that $\lambda(s)=0$ for $s \geqq \epsilon$ and $\lambda(s)=1$ for $s \leqq-\epsilon$. Define

$$
X \subset S^{2 k+1} \times S^{2 k+1} \times I
$$

to be the set of points $(x, y, 0)$ such that $d(x, y) \leqq \epsilon$ (where $d$ is the natural Riemannian metric of $\left.S^{2 k+1}\right)$ and

$$
X^{\prime} \subset S^{2 k+1} \times S^{2 k+1} \times I
$$

the set of points $\left(x, y, \lambda\left(x_{0}\right)\right)$ such that $d(x, \rho(y)) \leqq \epsilon$. Both $X$ and $X^{\prime}$ are diffeomorphic to the total space of the disk tangent bundle of $S^{2 k+1}$ and $X \cap X^{\prime}$ is a neighborhood of $(P, P, 0)$. Let $Y=X \cup X^{\prime}$. Define the involution $T: Y \rightarrow Y$ by

$$
T(x, y, t)=(\rho(x), \rho(y), t) .
$$

Then the only fixed points of $T$ are $(P, P, 0),(Q, Q, 0)$ and $(Q, Q, 1)$. After straightening the corners (this can be done in a way compatible with $T$ ), $Y$ becomes a differentiable manifold with boundary $\Sigma=\Sigma^{4 k+1}$ where $\Sigma$ is the Kervaire homotopy sphere and $T \mid \Sigma$ has no fixed points. However, since the presence of corners does not affect the value of $c(\Sigma, T)$ we shall continue to use the initial explicit description of $Y$ and of $\Sigma=\partial Y$.

Let $V \subset Y$ be the set of $(x, y, t) \in Y$ such that $x \in S^{2 k}$. Then $T V=V$ and $V$ is a $(4 k+1)$-manifold with $\partial V=N=\Sigma \cap V$. Let also $R$ $=\left\{(x, y, t) \mid x_{2 k+1} \geqq 0\right\}$. Then $Y=R \cup T R, V=R \cap T R$. Finally, if $A=\Sigma \cap R, B=\Sigma \cap T R$, then $\Sigma=A \cup B, N=A \cap B$, so that $N$ is a characteristic manifold for $(\Sigma, T)$.

Let $W \subset V$ be the set of $(x, y, t), x, y \in S^{2 k}$. Then $W$ consists of two copies of the tangent disk bundle of $S^{2 k}$, "plumbed" together in a neighborhood of $(P, P, 0)$. The only nonvanishing reduced homology group of $W$ is $H_{2 k}(W, Z)=Z+Z$; the two generators $e, f$ are represented by the imbeddings $S^{2 k} \rightarrow W$ by $(x, x, 0)$ and $\left(x, \rho(x), \lambda\left(x_{0}\right)\right)$, $x \in S^{2 k}$. The intersection coefficients are

$$
e \cdot e=f \cdot f=2, \quad e \cdot f=1 .
$$

For $y \in S^{2 k+1}$ such that $d\left(S^{2 k}, y\right) \leqq \epsilon$, let $p(y) \in S^{2 k}$ be the point in which the great circle through $y$ orthogonal to $S^{2 k}$ meets $S^{2 k}$. Let $W^{\prime} \subset \partial V=N$ be the set of $(x, y, t) \in N$ with $y_{2 k+1} \geqq 0$ and $W^{\prime \prime} \subset N$ the set of $(x, y, t) \in N$ with $y_{2 k+1} \leqq 0$. Then the correspondence $(x, y, t)$ $\rightarrow(x, p(y), t)$ is a homeomorphism of $W^{\prime}$ and of $W^{\prime \prime}$ onto $W$. More- 
over, $N=W^{\prime} \cup W^{\prime \prime}, \partial W=W^{\prime} \cap W^{\prime \prime}$ and $T W^{\prime}=W^{\prime \prime}$. In other words $N$ is the double of $W$ and $T$ maps one copy of $W$ onto the other.

3. Computation of $c(\Sigma, T)$. Lefschetz duality and the exactness of the homology sequence of the pair $(W, \partial W)$, together with (11), imply that $H_{i}(\partial W, Z)=0, i \neq 0,2 k-1,4 k-1$ and that $H_{2 k-1}(\partial W)=Z_{3}$. Therefore the inclusion

$$
j: H_{2 k}\left(W^{\prime}, Z_{2}\right) \oplus H_{2 k}\left(W^{\prime \prime}, Z_{2}\right) \stackrel{\approx}{\rightarrow} H_{2 k}\left(N, Z_{2}\right)=G
$$

is an isomorphism (which preserves intersections). By (11) and (12), $G$ has a basis $e^{\prime}, f^{\prime}, T e^{\prime}, T f^{\prime}$ represented by $e, f$ in $W^{\prime}$ and their images by $T$ in $W^{\prime \prime}$ and

$$
A\left(e^{\prime}, f^{\prime}\right)=1, \quad A\left(e^{\prime}, e^{\prime}\right)=A\left(f^{\prime}, f^{\prime}\right)=0
$$

and clearly

$$
B\left(e^{\prime}, f^{\prime}\right)=B\left(e^{\prime}, e^{\prime}\right)=B\left(f^{\prime}, f^{\prime}\right)=0 .
$$

Moreover, since $\frac{1}{2} e \cdot e=\frac{1}{2} f \cdot f=1$,

$$
\mu_{0}\left(e^{\prime}\right)=\mu_{0}\left(f^{\prime}\right)=1,
$$

whereas (3) implies that

$$
\psi_{0}\left(e^{\prime}\right)=\psi_{0}\left(f^{\prime}\right)=0 .
$$

As a consequence of (13) and (14) the images $\bar{e}, \bar{f} \in G_{T}$ of $e^{\prime}, f^{\prime}$ form a symplectic basis of $G_{T}$ with respect to $C$ and (15) and (16) imply that $\mu_{T}(\bar{e})=\mu_{T}(\bar{f})=1$ so that by $(10)$

$$
c(\Sigma, T)=c_{T}=1 \text {. }
$$

REMARK 1. Since $b P_{6}=b P_{14}=0$ [4], [6] and also $b P_{30}=0$ (by the recent work of Browder), there are fixed point free involutions with Arf invariant 1 on $S^{5}, S^{13}$ and $S^{29}$.

REMARK 2. It would be interesting to know what the relation is between the $T: S^{5} \rightarrow S^{5}$ constructed in this paper and the nonstandard involution on $S^{5}$ described in [2]. However, it follows from [8] that our involution is equivalent to a generator of the group $Z_{4}$ of fixed point free involutions on $S^{5}$.

After this paper has been completed, the author learned that the existence of differentiable involutions on a homotopy sphere $\Sigma^{4 k+1}$ for all $k$ with $c(\Sigma, T) \neq 0$ has been proven by an entirely different method by W. Browder (not yet published). Such an example on $\Sigma^{9}$ was also obtained by D. Sullivan. 
Added in proof. A PL-classification of fixed point free involutions analogous to that of [8], which also implies the existence of PLinvolutions with $c(\Sigma, T)=0$ has been obtained independently by S. López de Medrano (to appear in the Proceedings of the Tulane Conference on Transformation Groups, 1967).

\section{REFERENCES}

1. W. Browder and G. R. Livesay, Fixed point free involutions on homotopy spheres, Bull. Amer. Math. Soc. 73 (1967), 242-245.

2. M. W. Hirsch and J. W. Milnor, Some curious involutions of spheres, Bull. Amer. Math. Soc. 70 (1964), 372-377.

3. M. Kervaire, $A$ manifold which does not admit any differentiable structure, Comment. Math. Helv. 34 (1960), 257-270.

4. M. Kervaire and J. W. Milnor, Groups of homotopy spheres. I, Ann. of Math. 77 (1963), 504-537.

5. S. L6pez de Medrano, Involutions on homotopy spheres and homology 3-spheres, Bull. Amer. Math. Soc. 73 (1967), 727-731.

6. J. W. Milnor, Differentiable manifolds which are homotopy spheres, Mimeographed notes, Princeton University, Princeton, N. J., 1959.

7. C. T. C. Wall, Surgery on non-simply connected manifolds, Ann. of Math. 84 (1966), 216-276.

8. - Free piecewise linear involutions on spheres, Bull. Amer. Math. Soc. 74 (1968), 554-558.

CORNell UNIVERsity 